\title{
Effect of peritoneal dialysis vs. haemodialysis on respiratory mechanics in acute kidney injury patients
}

\author{
Cibele Puato Almeida ${ }^{1} \cdot$ André Luís Balbi $^{1} \cdot$ Daniela Ponce $^{1}$
}

Received: 23 January 2018 / Accepted: 1 June 2018 / Published online: 11 June 2018

(c) Japanese Society of Nephrology 2018

\begin{abstract}
Background Peritoneal dialysis (PD) and hemodialysis (HD) are options for the treatment of acute kidney injury (AKI) patients. The aim of this study was to compare the effects of PD and daily HD on respiratory mechanics of AKI patients undergoing invasive mechanical ventilation (IMV).

Methods A prospective cohort study evaluated 154 patients, 37 on continuous PD and 94 on HD. Respiratory mechanics parameters such as pulmonary static compliance (Psc) and resistance of the respiratory system (Rsr) and oxygenation index (OI) were assessed for 3 days. Patients were evaluated at moments 1, 2 and 3 (pre- and post-dialysis).

Results The initial clinical parameters were similar in the two groups, except the age that was higher in continuous PD group $(70.8 \pm 11.6$ vs. $60 \pm 15.8 ; p<0.0001)$. In both groups, Psc increased significantly, with no difference between the two groups-pre-dialysis (continuous PD 40 $\pm 17.4,42.8 \pm 17.2,48 \pm 19$; HD 39.1 $\pm 21.3,39.5 \pm 18.9$, 45.2 \pm 21 ) and postdialysis (continuous PD 42.8 $\pm 7.2,48 \pm 19,57.1 \pm 18.3$; HD 42 $\pm 19,45 \pm 18.5,56 \pm 24.8$ ). Rsr remained stable among patients on continuous PD (pre-dialysis $10.4 \pm 5.1,13.3 \pm 7.7,13.5 \pm 10.3$, post-dialysis $13.3 \pm 7.7,13.5 \pm 10.3,11.1 \pm 5.9$ ) and decreased among HD patients (pre-dialysis 10.4 $\pm 5.1,10.4 \pm 5.1,10.4 \pm 5,1$, post-dialysis 10.5 $\pm 6.8,10 \pm 4.9,8.9 \pm 4.2$ ). There was difference in Rsr between the two groups at the post-dialysis moments 1 and $2(p=0.03)$. OI increased in both groups (continuous PD 260.7 $\pm 119,252.7 \pm 87.1,287.3 \pm 88.4$; HD $228 \pm 85,257 \pm 84,312.1 \pm 111.5, p>0.05$ ), although there was no difference between them.

Conclusion AKI patients undergoing IMV and HD or PD had improvement in the mechanical ventilation and oxygenation, with no difference between the two groups.
\end{abstract}

Keywords Acute kidney injury $\cdot$ Continuous peritoneal dialysis $\cdot$ Daily hemodialysis $\cdot$ Respiratory mechanics · Oxygenation and invasive mechanical ventilation

\section{Introduction}

Acute kidney injury (AKI) is a common disorder that affects approximately $50 \%$ of patients in intensive care units [1]. The kidneys and lungs are the organs most often involved in critically ill patients, and the association between AKI and acute lung injury (ALI) may result in a mortality rate $>80 \%$ [2].

There is no consensus in the literature regarding the best dialysis method for the treatment of AKI. Intermittent haemodialysis (IHD) and continuous peritoneal dialysis (PD)

Daniela Ponce

dponce@fmb.unesp.br

1 Department of Internal Medicine, Botucatu Medical School, UNESP, Univ Estadual Paulista, Botucatu, Brazil are viable options. Recent studies have shown that PD can promote adequate metabolic and fluid control in some AKI patients, with a mortality rate similar to that of other dialysis modalities [3-9]; however, after infusion of the dialysate, PD can increase intra-abdominal pressure (IAP) and lead to impaired diaphragm mobilization, reduced inspiration and expiration pressure, reduced total pulmonary capacity and reduced functional residual capacity, which could cause or worsen respiratory failure [10-14].

Despite the important association between AKI and lung injury in patient prognosis, few studies have evaluated the influence of PD and HD on respiratory mechanics in AKI patients. Therefore, this study aimed to evaluate and compare respiratory mechanics, and oxygenation in mechanically ventilated AKI patients undergoing high-volume PD and IHD. 


\section{Methodology}

This prospective cohort study evaluated respiratory mechanics during high-volume PD or IHD in AKI patients undergoing mechanical ventilation. Patients were admitted to the Clinical Hospital of Botucatu Medical School over 18 consecutive months. The study was approved by the medical ethics committee for local research (protocol number 520362), and informed consent was obtained from all participants or their legal representatives.

Patients were included in the study if they were $>18$ years of age; had a clinical diagnosis of AKI according to AKI Network criteria [14], with AKI caused by ischemic, nephrotoxic or mixed acute tubular necrosis; were treated with at least one dialysis session and were using mechanical ventilation.

The timing for renal replacement therapy (RRT) was based on the concept of demand capacity balance, which was originally described by Macedo and Mehta [15]. We also started RRT when the patients had hypervolemia refractory to high dose diuretics, rapidly increasing hyperkalemia, severe refractory acidosis and symptoms/signs of uremia.

Exclusion criteria were pre- and post-renal AKI causation, hemodynamic instability (systolic blood pressure $<80 \mathrm{mmHg}$ or norepinephrine use at doses $>0.3 \mu \mathrm{g} / \mathrm{kg} / \mathrm{min}$ ), absolute contraindications for PD or IHD use, early mechanical complications related to PD (within $24 \mathrm{~h}$ ), pregnancy, severe chronic kidney disease (baseline serum creatinine $>4 \mathrm{mg} / \mathrm{dl}$ ), kidney transplantation, tracheostomy (where measurements of respiratory mechanics are not possible due to lack of deep sedation) and use of alveolar recruitment with positive end-expiratory pressure $>10 \mathrm{cmH}_{2} \mathrm{O}$.

\section{Dialysis methods}

Based on Kidney Disease Improving Global Outcomes (KDIGO) Clinical Practice Guidelines for Acute Kidney Injury [16], the AKI group at Botucatu Medical School developed its protocol for choice of dialysis method and dialysis dose for AKI patients. CRRT, PIRRT or PD are indicated for hemodynamically unstable patients (noradrenaline use $>0.3 \mu \mathrm{cg} / \mathrm{kg} / \mathrm{min}$ ). In patients with acute brain injury or increased intracranial pressure due to intracranial hemorrhage, we recommend to use CRRT or PD.

Relative contraindications for PD use are recent abdominal surgery, adynamic ileus, intra-abdominal adhesions, peritoneal fibrosis, peritonitis and for the treatment of emergencies such as acute pulmonary edema or life-threatening hyperkalemia. In these situations, HD is the first option for treating these patients.
The dialysis dose prescribed was $\mathrm{Kt} / V=1.4$ for IHD and PIRRT treatment, 0.6 for PD and $30 \mathrm{ml} / \mathrm{kg} / \mathrm{h}$ for CRRT.

Patients were divided into two groups according to the dialysis method used: the PD group, with patients undergoing continuous high-volume PD, and the HD group, with patients undergoing daily IHD (not using noradrenaline or using it at doses $<0.3 \mu \mathrm{g} / \mathrm{kg} / \mathrm{min}$ ).

\section{HD}

The HD sessions lasted for 3-5 $\mathrm{h}$ and were held six times a week. A double lumen catheter was inserted blindly for central venous access (jugular or femoral vein) by a nephrologist at the bedside under local anaesthesia. Blood and dialysate flows were 300 and $500 \mathrm{ml} / \mathrm{min}$, respectively. A volumetric HD machine (Fresenius 4008F) and polysulfone dialyzer (FX 60 or 80 ) with a prescribed $\mathrm{Kt} / V$ of $1.4 /$ session were used. The concentrations of bicarbonate $(26-36 \mathrm{mEq} / \mathrm{l})$, potassium (1-3 mEq/l), sodium (140-145 $\mathrm{mEq} / \mathrm{l})$ and calcium $(2.5-3.5 \mathrm{mEq} / \mathrm{l})$ of the dialysis bath were adjusted according to the exams and individual needs of the patients. The ultrafiltration rate (UF) did not exceed $500 \mathrm{ml} / \mathrm{h}$ and the bath temperature ranged from 35 to $35.5^{\circ} \mathrm{C}$. During the sessions, the patients were anticoagulated with a 50-100 IU/kg bolus dose of heparin, and then with 500-1000 IU/h in the following hours. In cases of contraindication to anticoagulation, the system was washed with $50 \mathrm{ml}$ of $0.9 \%$ sodium chloride every $30 \mathrm{~min}$, throughout the entire procedure.

\section{PD}

A continuous high-volume PD was used as it was designed to achieve higher small-solute clearances. The modality used an automated cycler, a flexible catheter and a high volume of dialysis fluid as described previously [2-8]. Each highvolume PD session lasted $24 \mathrm{~h}$, and sessions were repeated daily. The prescribed $\mathrm{Kt} / V$ was $0.60 /$ session, and the total dialysate volume per session ranged from 36 to 441 (2 1/ cycle). Peritoneal access was established by the nephrology team who used a trocar to introduce a percutaneous Tenckhoff catheter. The dialysate used was Dianeal (Na, $132 \mathrm{mEq} / \mathrm{l} ; \mathrm{Ca}, 3.5 \mathrm{mEq} / \mathrm{l} ; \mathrm{K}, 0 \mathrm{mEq} / \mathrm{l} ; \mathrm{Mg}, 1.5 \mathrm{mEq} / \mathrm{l}$; lactate, $40 \mathrm{mEq} / \mathrm{l}$; glucose, 1.5, 2.5 or $4.25 \%$ ) (Baxter, Sao Paulo, Brazil), and the exchanges were performed using a Home Choice cycler (Baxter).

Respiratory mechanics, IAP and oxygenation were evaluated. Respiratory mechanics were evaluated at six time points over 3 days: M1 (before and after the first dialysis session), M2 (before and after the second dialysis session) and M3 (before and after the third dialysis session). Estimates of pulmonary compliance (Psc) and system resistance (Rsr) were based on the technique of end-inspiratory occlusion [17]. Such measures require the patients to be 
paralyzed to eliminate spontaneous inspiratory effort [18]. Therefore, patients who had not previously been sedated were temporarily sedated by a physician who evaluated and prescribed medication until they reached deep levels of sedation ( -5 on the Richmond Agitation Sedation Scale [19]) and the spontaneous inspiratory effort was absent. Patients were paralyzed, if necessary. These measurements were performed in the presence of physicians of ICU, who prescribed the drugs.

Patients were ventilated using a constant-flow (squarewave) volume-controlled mode with an inspiratory pause of at least $2 \mathrm{~s}$, a positive end-expiratory pressure of $5 \mathrm{cmH}_{2} \mathrm{O}$ and a tidal volume of $6 \mathrm{ml} / \mathrm{kg}$ (ideal weight). Five consecutive measures of respiratory pressure peak, plateau pressure, tidal volume, inspiratory flow, and autopositive end-expiratory pressure were collected at each assessment, and average values were used for the calculations [20].The efficiency of gas exchange was assessed daily (pre- and post-dialysis) by an oxygenation index, corresponding to the ratio of the arterial oxygen pressure to the fraction of inspired oxygen $\left[\mathrm{PaO}_{2} / \mathrm{FiO}_{2}(20)\right]$, and by the fraction of $\mathrm{FiO}_{2}$ (expressed as a percentage).

An indirect technique, based on the method of Kron et al., using a three-way intravesical catheter was used to measure IAP [21]. Saline $(25 \mathrm{ml})$ was instilled into the bladder, and the patient was leveled into a completely supine position (zero reference point at the mid-axillary line) as determined by the International Conference of Experts on Intra abdominal Hypertension and Abdominal Compartment Syndrome [22]. After saline infusion, the pressure monitor was expected to be stabilized at a fixed value to ensure its reliability [23, 24].

The study protocol consisted of general data and the assessment of respiratory mechanics, oxygenation, ultrafiltration (UF) during each dialysis session and 24-h fluid balance.

Data analysis was performed using SAS (version 9.2; SAS Institute, Cary, NC, USA). Variables with normal distribution were described using the mean \pm standard deviation, while variables with non-normal distribution were described using medians and interquartile ranges. Repeated-measures analysis of variance and multiple comparisons with Tukey's adjustment were used to analyse Rsr and $\mathrm{PaO}_{2} / \mathrm{FiO}_{2}$. Comparisons of Psc, IAP, and $\mathrm{FiO}_{2}$ were performed using a repeated-measures model and asymmetric distribution (gamma) under the GENMOD procedure. Multiple-comparison tests were performed by the same procedure using the DIFF option. The same type of corrected adjustment was used for age, patients with chronic obstructive pulmonary disease, pulmonary infection and invasive mechanical ventilation (IMV) time. The level of significance was $5 \%$ in all statistical tests.

\section{Results}

During the study period, 154 patients received dialysis treatment: 45 by PD (29.2\%) and 109 by IHD (70.8\%). Absolute contraindications for PD were present in 71 patients $(46.1 \%)$ and included recent abdominal surgery ( $<1$ month), multiple abdominal surgeries (more than three procedures), severe hyperkalemia with electrocardiography changes, severe respiratory failure $\left(\mathrm{FiO}_{2}>70 \%\right)$ and severe fluid overload. Of the 45 patients treated with PD, eight (17.8\%) were excluded from the analyses as they experienced mechanical complications related to the peritoneal catheter in the first $24 \mathrm{~h}$ of dialysis $(n=4)$ or underwent a tracheostomy $(n=4)$. Of the 109 patients treated with HD, 15 were excluded as they presented higher hemodynamic instability during the first IHD session. They were subsequently changed to prolonged HD. Therefore of the remaining 131 AKI patients, 37 were treated by PD and 94 by IHD. This represented 111 sessions of high-volume PD and 282 sessions of IHD (a total of three dialysis sessions were analysed for all patients).

The clinical characteristics of the patients studied at the first evaluation and the chosen modality of dialysis are described in Table 1. The groups were similar for all characteristics except age, which was higher in the PD group $(70.8 \pm 11.6$ vs. $60 \pm 15.8, p<0.0001)$.

\section{Assessment of respiratory mechanics}

The parameters of respiratory mechanics were compared between the two groups at the pre- (1, 2 and 3$)$ and postdialysis time points (1, 2 and 3 ). Table 2 shows the results of Psc at the pre- and post-dialysis time points. There was no significant difference between the groups during the 3 days of evaluation.

There was no statistically significant difference in Rsr between the groups at the pre-dialysis time point; however, at post-dialysis, values in the HD group were smaller than in the PD group on days 1 and 2. These data are presented in Table 3.

Patients treated by PD showed an improved oxygenation index, although there was no statistically significant difference between the time points measured (first day $=260.7 \pm 119$, second day $=252.8 \pm 87.1$ and third day $=287.3 \pm 88.4)$. Patients treated with HD also showed an improved oxygenation index (first day $=228 \pm 85$, second day $=257 \pm 84$ and third day $=312.1 \pm 111.5$ ); however, these improvements were statistically different on the third day of evaluation compared to the preceding 2 days (third day vs. first day, $p=0.0001$; third day vs. second day, $p=0.0268$ ). There was no statistically significant difference between the PD and HD groups. 
Table 1 Clinical, laboratory and respiratory characteristics of acute kidney injury patients at the beginning of dialysis treatment

Table 2 Pulmonary compliance of AKI patients treated with daily IHD and PD

\begin{tabular}{llll}
\hline & Hemodialysis $(n=94)$ & $\begin{array}{l}\text { Peritoneal dialysis } \\
(n=37)\end{array}$ & $p$ \\
\hline Dialysis sessions $(n)$ & 282 & 111 & 0.26 \\
Male sex (\%) & $58(61.7)$ & $28(75.6)$ & $<0.0001$ \\
Age (years) & $60 \pm 15.8$ & $70.8 \pm 11.6$ & \\
Aetiology of AKI & & & 1.0 \\
Ischemic/septic & $79(84)$ & $31(82.3)$ & 1.0 \\
Mixed & $15(16)$ & $6(17.6)$ & 0.07 \\
Sepsis (\%) & $85(90.4)$ & $28(75.6)$ & 0.2 \\
Oliguria (\%) & $56(59.4)$ & $18(48.6) 5$ & 0.98 \\
Pneumonia (\%) & $17(18)$ & $7(18.9)$ & 0.92 \\
COPD (\%) & $8(8.5)$ & $4(10.8)$ & 0.82 \\
Mechanical ventilation time (days) & $14 \pm 4$ & $11 \pm 3$ & \\
Indication for dialysis (\%) & & & 0.21 \\
Uremia & $49(42.5)$ & $24(63.6)$ & 0.22 \\
Hypervolemia & $33(35.1)$ & $8(21.6)$ & 0.32 \\
Noradrenaline use (\%) & $75(79.2)$ & $26(70.3)$ & 0.41 \\
APACHE II & $24.7 \pm 4.5$ & $23.9 \pm 4.9$ & 0.74 \\
ATN-ISS & $0.66 \pm 0.16$ & $0.68 \pm 0.16$ & 0.16 \\
PaO $/$ FiO & $226.8 \pm 85.3$ & $260.7 \pm 117.1$ & \\
\hline
\end{tabular}

APACHE II Acute Physiological and Chronic Health Evaluation II, ATN-ISS Acute Tubular Necrosis-Individual Severity Score. Data expressed in absolute values, mean or median, COPD chronic obstructive pulmonary disease

\begin{tabular}{llcllll}
\hline Group & Day & Psc & Group & Day & Cest & $p$ \\
\hline Pre-dialysis & & & & & & \\
PD & 1 & $40 \pm 17.4$ & HD & 1 & $39.1 \pm 21.3$ & 0.8 \\
PD & 2 & $42.8 \pm 17.2$ & HD & 2 & $39.5 \pm 18.9$ & 0.35 \\
PD & 3 & $48 \pm 19$ & HD & 3 & $45.2 \pm 21$ & 0.54 \\
Post-dialysis & & $42.8 \pm 17.2$ & HD & 1 & & \\
PD & 1 & $48 \pm 19$ & HD & 2 & $42 \pm 19$ & 0.8 \\
PD & 2 & $57.1 \pm 18.3$ & HD & 3 & $56 \pm 24.8$ & 0.83 \\
PD & 3 & &
\end{tabular}

There was no statistically significant difference after adjusting for age, chronic obstructive pulmonary disease, pneumonia, and invasive mechanical ventilation time

$P D$ peritoneal dialysis, $H D$ hemodialysis, $P c s$ pulmonary compliance

\begin{tabular}{lllllll}
\hline Group & Day & Rsr & Group & Day & Rsr & $p$ \\
\hline Pre-dialysis & & & & & & \\
PD & 1 & $10.4 \pm 5.1$ & HD & 1 & $11.1 \pm 6.2$ & 0.48 \\
PD & 2 & $13.3 \pm 7.7$ & HD & 2 & $11.3 \pm 5.2$ & 0.14 \\
PD & 3 & $13.5 \pm 10.3$ & HD & 3 & $11.4 \pm 6$ & 0.26 \\
Post-dialysis & & $13.3 \pm 7.7$ & HD & 1 & $10.5 \pm 6.8$ & 0.03 \\
PD & 1 & $13.5 \pm 10.3$ & HD & 2 & $10 \pm 4$ & 0.03 \\
PD & 2 & $11.1 \pm 5.9$ & HD & 3 & $8.9 \pm 4.2$ & 0.07 \\
PD & 3 & &
\end{tabular}

There was no statistically significant difference after adjusting for age, chronic obstructive pulmonary disease, pneumonia, and invasive mechanical ventilation time

$P D$ peritoneal dialysis, $H D$ hemodialysis, $R s r$ respiratory System resistance 


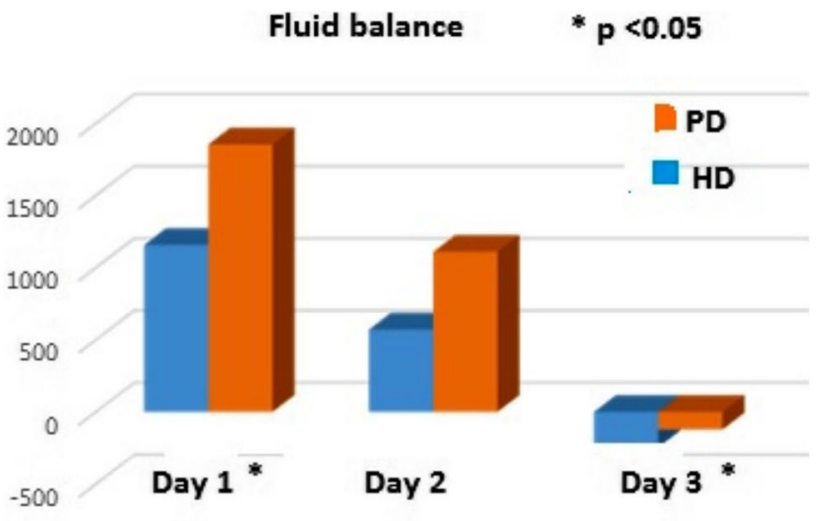

*FB day 1 vs FB day 3 for both (HD and PD) treatment- $<<0.05$

Fig. 1 Fluid balance during PD and HD treatment of AKI patients

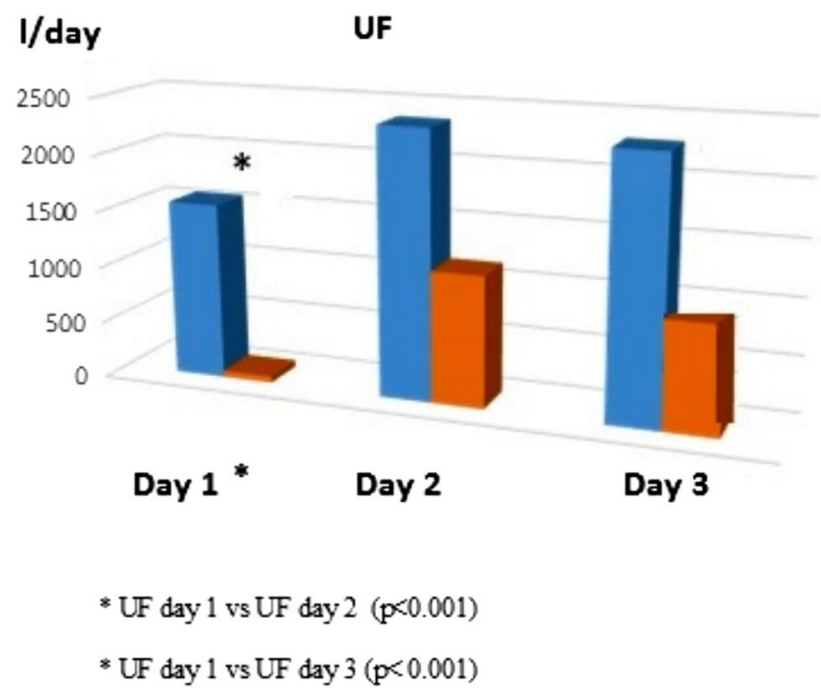

Fig. 2 Ultrafiltration during PD and HD treatment of AKI patients

In the patients treated with PD and $\mathrm{HD}$, fluid balance (FB) showed a progressive reduction, with a statistically significant difference between time points 3 and $1(p<0.05)$. In addition, UF presented a progressive increase on the second and third days $(p<0.05)$. Figures 1 and 2 show the daily comparative values of FB and UF, respectively, between the two groups. There was no difference in FB between the two groups at day
3, while UF was different between the two groups on all three evaluated days: UF was lower in the PD group.

IAP was evaluated only in patients treated by PD and there was no increase during the treatment (Table 4).

After adjustment for age, the presence or absence of chronic obstructive pulmonary disease, lung infection and IMV time, the results obtained for mechanical ventilation, respiratory peak pressure and oxygenation index did not change.

\section{Discussion}

The present study aimed to compare the effects of HD and PD on the respiratory function of AKI patients under IMV. The results suggest an improvement in respiratory mechanics and oxygenation with no differences between the two groups.

In relation to $\mathrm{PD}$, changes in pulmonary function may occur during and after dialysate infusion into the abdominal cavity, mainly due to the increase in IAP. An increase in abdominal and diaphragmatic pressure causes a change in pleural pressure and a decrease in pulmonary compliance and total lung capacity [24]. Bunchman et al. [25] evaluated PD in four children with AKI under IMV and found a reduction of Psc and an increase of Rsr during dialysate infusion. This worsening of the ventilatory mechanics was attributed to the increase in IAP. Morris et al. [26] evaluated different volumes of dialysate $(0,10,20$ and $30 \mathrm{ml} / \mathrm{kg})$ and found an increase in IAP with increasing dialysate volume; however, this was not associated with any deleterious effects on lung function.

Previous data from this research group suggested that patients with PD under IMV showed improved respiratory mechanics. IAP showed only a temporary increase in this study, returning to values very close to basal on the third day of dialysis, and there was no impairment in respiratory mechanics [27]. In the present study, a progressive increase was observed in Psc in patients treated with PD, reflecting an improvement in ventilatory mechanics. In addition, the Rsr remained constant and oxygenation improved in absolute values. This finding may be related to the UF that was able to significantly reduce $\mathrm{FB}$ during the dialytic procedure. Clinical data suggest that positive FB and oliguria negatively contribute to the pulmonary prognosis [28-31].

Table 4 Intra-abdominal pressure of AKI patients treated by peritoneal dialysis

\begin{tabular}{|c|c|c|c|c|c|c|c|}
\hline & $\begin{array}{l}\text { Pre-dialysis } \\
1\end{array}$ & $\begin{array}{l}\text { Post-dialysis } \\
1\end{array}$ & $\begin{array}{l}\text { Pre-dialysis } \\
2\end{array}$ & $\begin{array}{l}\text { Post-dialysis } \\
2\end{array}$ & $\begin{array}{l}\text { Pre-dialysis } \\
3\end{array}$ & $\begin{array}{l}\text { Post-dialysis } \\
3\end{array}$ & $p$ \\
\hline IAP $(\mathrm{mmHg})$ & $8.9 \pm 4.4$ & $10.1 \pm 5.3$ & $10.2 \pm 5.9$ & $9.6 \pm 4.2$ & $8.8 \pm 4.9$ & $9.2 \pm 4.3$ & $>0.05$ \\
\hline
\end{tabular}


Werner et al. [31] evaluated PD in 32 children with AKI and found a reduction in mean airway pressure. They suggested that PD may be an effective method for withdrawing fluid and consequently reducing pulmonary oedema. Bolkariia et al. [32] also found improved lung function in children with AKI after 4 days of PD.

With regards to HD, this study also showed an improvement in ventilatory mechanics and oxygenation. The accumulation of fluid around small airways results in premature closure and air entrapment and leads to increased respiratory work. These changes decrease the pulmonary compliance and increase the airway resistance [33]. Thus, it is possible that the improvement in pulmonary function found in the present study was a result of a reduction of pulmonary oedema achieved through fluid withdrawal by UF during the HD sessions.

Huang et al. [34] evaluated 14 patients with AKI and chronic kidney disease and observed an improvement in Rsr and dynamic complacency, which they attributed to the UF achieved. The authors suggested that the negative balance produced by haemodialysis could lead to a decrease in peribronchial oedema. However, Stein et al. [35] suggested that the removal of pulmonary oedema alone was not sufficient to explain the improved lung function. Although not analysed in the present study, it is likely that the withdrawal of inflammatory mediators during HD may also have an influence on respiratory function. Interleukins 6 and 8 appear to play a key role in the development of acute pulmonary injury, and their molecular weight (21 and $8 \mathrm{kDa}$, respectively) is below the cut-off of HD 48 filters.

In an experimental model of ALI, Lu et al. [36] evaluated the effects of continuous venovenous hemodiafiltration on the pulmonary function of pigs and found improved ventilatory mechanics and oxygenation after $6 \mathrm{~h}$ of dialysis. They suggested that the improved lung function with HD was due to the reduction of both pulmonary oedema and inflammatory mediators (i.e., TNF- $\alpha$ ). Likewise, in a prospective clinical study, Lopes et al. [33] showed increased Psc in patients with AKI and CKD after HD, regardless of the reduction in blood volume. Conversely, Steinhorst et al. [37] and Huang et al. [38] evaluated the ventilatory mechanics in patients submitted to HD under IMV and did not find any significant alterations.

There are reports in the literature that relate HD with hypoxemia via several mechanisms, including via the activation of the complement system. The contact between blood and the extracorporeal circuit can lead to neutrophil stimulation and cause leukocyte accumulation in the lungs with consequent hypoxemia [39]; however, this type of problem can be solved with the use of more biocompatible membranes, such as polysulfone used in this study. Hypoxemia may also be explained by decreased alveolar ventilation in response to the diffusion of $\mathrm{CO}_{2}$ within the dialysate. As a result, the $\mathrm{CO}_{2}$ content in venous blood reduces and decreases the central ventilatory stimulus and minute ventilation. As alveolar ventilation decreases and $\mathrm{O}_{2}$ extraction remains the same, $\mathrm{PaO}_{2}$ also decreases [40]. However, the present study showed improved gas exchange with a significant increase in oxygenation. These results suggest that the redistribution of pulmonary ventilation after volume withdrawal by UF allows the ventilation of alveoli that were previously filled with liquids.

Another hypothesis that can be considered for the improved respiratory mechanics in patients with CPD submitted to HD is the removal of uremic toxins by the dialysis method. Harper et al. [40] evaluated patients with CKD and found a significant increase in pulmonary vascular permeability to water and proteins in the presence of uremic serum. Increased vascular permeability may lead to oedema formation and this may interfere with pulmonary expansion.

When comparing lung function in patients undergoing PD and HD, this study showed similar results. There was no difference in Psc between groups at any of the time points, preor post-dialysis. Oxygenation also showed similar results between the groups at the three time points. Rsr showed a significant difference in the first two post-dialysis sessions, with more satisfactory results among those who underwent HD. However, Rsr may be influenced by punctual factors (i.e., bronchospasm, the presence of secretions, the condensation of liquids in the mechanical respirator system, etc.) which makes it a less adequate tool than Psc for assessing the pulmonary parenchyma.

The main limitation of this study was the inability to randomize patients to the PD and HD groups. Even so, the groups were similar in several clinical and laboratory aspects.

In conclusion, the results presented here suggest that the use of PD and daily HD in patients with AKI under IMV improves ventilatory mechanics and oxygenation, but that there is no difference between the groups.

\section{Compliance with ethical standards}

Conflict of interest The authors have declared that no conflict of interest exists.

\section{References}

1. Hoste EA, et al. Epidemiology of acute kidney injury in critically ill patients: the multinational AKI_EPI study. Intensive Care Med. 2015;41:1411-23.

2. Ko GJ, Tabb H, Hassoun HT. Kidney-lung crosstalk in critically ill patient. Blood Purif. 2009;28:75-83.

3. Ponce D, Caramori JCT, Balbi AL, Barretti P. Peritoneal dialysis in acute kidney injury: Brazilian experience. Perit Dial Int. 2012;32:242-3. 
4. Ponce D, Berbel MN, Regina de Goes C, Almeida CTP, Balbi AL. High-volume peritoneal dialysis in acute kidney injury: indications and limitations. Clin J Am Soc Nephrol. 2012;7(6):887-94.

5. Gabriel DP, Balbi AL, Amerling R. Advances in peritoneal dialysis in acute kidney injury. Blood Purif. 2012;34:107-16.

6. Ponce D, Berbel MN, Abrão JMG, Goes CR, Balbi AL. A randomized clinical trial of high-volume peritoneal dialysis versus extended daily hemodialysis for acute kidney injury patients. Int Urol Nephrol. 2012;1:1-10.

7. Ponce D, Balbi AL. Peritoneal dialysis in acute kidney injury: a viable alternative. Perit Dial Int. 2011;31:387-9.

8. Goes CR, Berbel MN, Balbi AL, Ponce D. Metabolic implications of peritoneal dialysis in acute kidney injury patients. Perit Dial Int. 2013 (accepted for publication).

9. Gavelli G, Zompatori. Thoracic complications in uremic patients and in patients undergoing dialytic treatment: state of the art. Eur Radiol. 1997;7:708-17.

10. Hughes GC, Ketchersid TL, Lenzen JM, Lowe JE. Thoracic complications of peritoneal dialysis. Ann Thorac Surg. 1999;67:1518-22.

11. Mahale AS, Katyal A, Khanna R. Complications of peritoneal dialysis related to increased intra-abdominal pressure. Adv Perit Dial. 2003;19:130-5.

12. Pelosi $\mathrm{P}$, Quintel M, Malbrain ML. Effect of intra-abdominal pressure on respiratory mechanics. Acta Clin Belg. 2007;62(1):78-88.

13. Toens CH, Schachtrupp A, Hoer J, Junge K, Klosterhalfen B, Schumpelick V. Porcine model of abdominal compartment syndrome. Shock. 2002;18:37-45.

14. Mehta RL, Kellum JA, Shah SV, Molitoris BA, Ronco C, Warnock DG, et al. Acute Kidney Injury Network (AKIN): report of an initiative to improve outcomes in acute kidney injury. Crit Care. 2007;11:31-8.

15. Macedo E, Mehta RL. When should renal replacement therapy be initiated for acute kidney injury? Semin Dial. 2011;24:132-7.

16. KDIGO kidney disease improving global outcomes. Clinical practice guideline for acute kidney. Kidney Int Suppl 2012;2:1e138.

17. Levy P, Similowski T, Corbeil C, et al. A method for studying the static volume-pressure curves of the respiratory system during mechanical ventilation. J Crit Care. 1989;4:83-9.

18. Grinnan DC, Truwit JD. Clinical review: respiratory mechanics in spontaneous and assisted ventilation. Crit Care. 2005;9:472-84.

19. Ely EW, Truman B, Shintani A, Thomason JW, Wheeler AP, Gordon S, et al. Monitoring sedation status over time in ICU patients-reliability and validity of the Richmond AgitationSedation Scale (RASS). JAMA. 2003;289:2983-91.

20. Gilbert R, Keighley JF. The arterial/alveolar oxygen tension ratio. An index of gas exchange applicable to varying inspired oxygen concentrations. Am Rev Respir Dis. 1974;109:142.

21. Kron IL, Harman PK, Nolan SP. Measurement of intra-abdominal pressure as a criterion for abdominal re-exploration. Ann Surg. 1984;199:28-30.

22. Cheatham ML, Malbrain ML, Kirkpatrick A, Sugrue M, Parr M, De Waele J, et al. Results from the international conference of experts on intra-abdominal hypertension and abdominal compartment syndrome. II. Recommendations. Intensive Care Med. 2007;33:951-62.
23. Malbrain ML, Chiumello D, Pelosi P, Bihari D, Innes R, Ranieri $\mathrm{VM}$, et al. Incidence e prognosis of intra-abdominal hypertension in a mixed population of critically ill patients. A multiple-center epidemiology study. Crit Care Med. 2005;33(2):315-22.

24. Torquato J, Lucato JJJ, Antunes T, Barbas CV. Interaction between intra-abdominal pressure and positive-end expiratory pressure. Clinics. 2009;64(2):105-12.

25. Bunchman TE, et al. Pulmonary function variation in ventilator dependent critically ill infants on peritoneal dialysis. Adv Perit Dial. 1992;8:75-8.

26. Morris KP, Butt WW, Karl TR. Effect of peritoneal dialysis on intra-abdominal pressure and cardio-respiratory function in infants following cardiac surgery. Cardiol Young. 2004;14(3):293 -98 .

27. Almeida CP, Ponce D, De Marchi AC, Balbi AL. Effect of peritoneal dialysis on respiratory mechanics in acute kidney injury patients. Perit Dial Int. 2014;34(5):544-9.

28. Lewis CA, Martin GS. Understanding and managing fluid balance in patients with acute lung injury. Curr Opin Crit Care. 2004;10(1):13-7.

29. Prowle JR, Echeverri JE, Ligabo EV, et al. Fluid Balance and acute kidney injury. Nat Rev Nephrol. 2010;6:107-15.

30. Payen D, Pont AC, Sakr Y, et al. A positive fluid balance is associated with a worse outcome in patients with acute renal failure. Crit Care. 2008;12:74-80.

31. Werner HA, Wensley DF, Lirenman DS, et al. Peritoneal dialysis in children after cardiopulmonary bypass. J Thorac Cardiovasc Surg. 1997;113:64-70.

32. Bokariia LA, et al. Peritoneal dialysis in the newborn and infants after radical correction of complex congenital heart defects. Anestesiol Reanimol. 2002;2:42-8.

33. Lopes FM, Ferreira JR, Flores DG. Impacto da terapia renal substitutiva na função respiratória de pacientes sob ventilação mecânica. Rev bras ter intensiva. 2013;25(3):1-10.

34. Huang CC, et al. Respiratory drive and pulmonary mechanics during haemodialysis with ultrafiltration in ventilated patients. Anaesth Intensive Care. 1997;25:464-70.

35. Stein B, Pfenninger E, Guünert A, et al. The consequences of continuous haemofiltration on lung mechanics and extravascular lung water in a porcine endotoxic shock model. Intensive Care Med. 1991;17(5):293-8.

36. Lu GP, et al. Effect of continuous veno-venous hemodiafiltration on endotoxin-induced acute lung injury of the piglets. Pediatr Crit Care Med. 2011;12(2):73-8.

37. Steinhorst RC, Vieira JM, Abdulkader RCRM.. Acute effects of intermittent hemodialysis and sustained low-efficiency hemodialysis (SLED) on the pulmonary function of patients under mechanical ventilation. Renal Fail. 2007;29:341-5.

38. Huang CC, Lin MC, Yang CT, et al. Oxygen, arterial blood gases and ventilation are unchanged during dialysis in patients receiving pressure support ventilation. Respir Med. 1998;92:534-40.

39. Pierson DJ. Respiratory considerations in the patient with renal failure. Respir Care. 2006;51(4):413-22.

40. Harper SJ, Tomson CRV, Bates DO. Human uremic plasma increases microvascular permeability to water and protein in vivo. Kidey Int. 2002;61:1416-22. 